\title{
A Search Method for Large Polarization Kernels
}

\author{
Grigorii Trofimiuk \\ ITMO University, Russia \\ Email: gtrofimiuk@itmo.ru
}

\begin{abstract}
A new search method for large polarization kernels is proposed. The algorithm produces a kernel with given partial distances by employing depth-first search combined with some methods for search space reduction. Using the proposed method, we improved almost all existing lower bounds on the maximum rate of polarization for kernels of size from 17 to 27 .

We also obtained kernels which admit low complexity processing by the recently proposed recursive trellis algorithm. Numerical results demonstrate the advantage of polar codes with the proposed kernels compared with shortened polar codes and polar codes with small kernels.
\end{abstract}

\section{INTRODUCTION}

Polar codes are a novel class of error-correcting codes, which achieves the symmetric capacity of a binary-input memoryless channel $W$. They have low complexity construction, encoding, and decoding algorithms [1].

Polarization is a general phenomenon and it is not restricted to the Arikan matrix [2]. One can replace it by a larger matrix, called polarization kernel, which has better polarization properties, thus obtaining better finite length performance. Polar codes with large kernels were shown to provide an asymptotically optimal scaling exponent [3]. Some fundamental limitations on the rate of polarization together with various kernel constructions were proposed in [2], [4], [5].

The rate of polarization of the kernel depends only on its partial distances. In this paper, we propose a search algorithm for polarization kernels with given partial distances. The proposed approach is based on depth-first search combined with some methods for search space reduction. This allows us to find polarization kernels of size $17 \leq l \leq 27$ (except $l=24$ ) with better rate of polarization compared with the ones constructed in [2], [4].

We also demonstrate the application of the proposed approach to the construction of polarization kernels which admit low complexity processing by the recently proposed recursive trellis algorithm [6], [7]. Simulation results show that polar codes with the obtained large kernels provide significant performance gain compared with shortened polar codes and polar codes with small kernels.

\section{BACKGROUND}

\section{A. Notations}

For a positive integer $n$, we denote by $[n]$ the set of $n$ integers $\{0,1, \ldots, n-1\}$. The vector $u_{a}^{b}$ is a subvector $\left(u_{a}, u_{a+1}, \ldots, u_{b}\right)$ of some vector $u$. For vectors $a$ and $b$ we denote their concatenation by a.b. $M[i]$ is the $i$-th row of the matrix $M$. By $M[i, j]$ we denote the $j$-th element of $M[i]$.
For matrices $A$ and $B$ we denote their Kronecker product by $A \otimes B$. By $A^{\otimes m}$ we denote $m$-fold Kronecker product of matrix $A$ with itself.

\section{B. Polarizing transformation}

A polarization kernel $K$ is a binary invertible $l \times l$ matrix, which is not upper-triangular under any column permutation [2]. An $(n, k)$ mixed-kernel polar code [8], [9] is a linear block code generated by $k$ rows of matrix $G_{n}=\mathcal{K}_{1} \otimes \mathcal{K}_{2} \otimes \ldots \otimes \mathcal{K}_{s}$, where $\mathcal{K}_{i}$ is an $l_{i} \times l_{i}$ polarization kernel and $n=\prod_{i=1}^{s} l_{i}$. The encoding scheme is given by $c_{0}^{n-1}=u_{0}^{n-1} G_{n}$, where $u_{i}, i \in \mathcal{F}$ are set to some pre-defined values, e.g. zero (frozen symbols), $|\mathcal{F}|=n-k$, and the remaining values $u_{i}$ are set to the payload data.

\section{Fundamental parameters of polar codes}

1) Rate of polarization: Let $W:\{0,1\} \rightarrow \mathcal{Y}$ be a symmetric binary-input discrete memoryless channel (B-DMC) with capacity $I(W)$. By definition,

$$
I(W)=\sum_{y \in \mathcal{Y}} \sum_{x \in\{0,1\}} \frac{1}{2} W(y \mid x) \log \frac{W(y \mid x)}{\frac{1}{2} W(y \mid 0)+\frac{1}{2} W(y \mid 1)} .
$$

The Bhattacharyya parameter of $W$ is

$$
Z(W)=\sum_{y \in \mathcal{Y}} \sqrt{W(y \mid 0) W(y \mid 1)} .
$$

Consider the polarizing transform $K^{\otimes m}$, where $K$ is an $l \times l$ polarization kernel, and bit subchannels $W_{m}^{(i)}\left(y_{0}^{n-1}, u_{0}^{i-1} \mid u_{i}\right)$, induced by it. Let $Z_{m}^{(i)}=Z\left(W_{m}^{(i)}\left(y_{0}^{n-1}, u_{0}^{i-1} \mid u_{i}\right)\right)$ be a Bhattacharyya parameter of $i$-th subchannel, where $i$ is uniformly distributed on the set $\left[l^{m}\right]$. Then, for any B-DMC $W$ with $0<I(W)<1$, we say that an $l \times l$ matrix $K$ has the rate of polarization (also known as error exponent) $E(K)$ if [2]

(i) For any fixed $\beta<E(K)$,

$$
\liminf _{n \rightarrow \infty} \operatorname{Pr}\left[Z_{n} \leq 2^{-l^{n \beta}}\right]=I(W) .
$$

(ii) For any fixed $\beta>E(K)$,

$$
\liminf _{n \rightarrow \infty} \operatorname{Pr}\left[Z_{n} \geq 2^{-l^{n \beta}}\right]=1 \text {. }
$$

Suppose we construct $(n, k)$ polar code $\mathcal{C}$ with kernel $K$. Let $P_{e}(n)$ be a block error probability of $\mathcal{C}$ under transmission over $W$ and decoding by SC algorithm. It was proven [2], that if $n / k<I(W)$ and $\beta<E(K)$, then for sufficiently large $n$ the probability $P_{e}(n)$ can be bounded as $P_{e}(n) \leq 2^{-n^{\beta}}$. 
It turns out that the rate of polarization is independent of channel $W$. Namely, let $\left\langle g_{1}, g_{2}, \ldots, g_{k}\right\rangle$ be a linear code, generated by vectors $g_{1}, g_{2}, \ldots, g_{k}$. Let $d_{H}(a, b)$ be the Hamming distance between $a$ and $b$. Let $d_{H}(b, \mathcal{C})=\min _{c \in \mathcal{C}} d_{H}(b, c)$ be a minimum distance between vector $b$ and linear block code $\mathcal{C}$. Let $\mathrm{wt}(b)=d_{H}(b, \mathbf{0})$.

The partial distances(PD) $\mathcal{D}_{i}, i=0, \ldots, l-1, l \times l$ of the kernel $K$ are defined as follows:

$$
\begin{aligned}
\mathcal{D}_{i} & =d_{H}(K[i],\langle K[i+1], \ldots, K[l-1]\rangle), i \in[l-1], \\
\mathcal{D}_{l-1} & =d_{H}(K[l-1], \mathbf{0}) .
\end{aligned}
$$

The vector $\mathcal{D}$ is referred to as a partial distances profile (PDP). It was shown in [2] that for any B-DMC $W$ and any $l \times l$ polarization kernels $K$ with PDP $\mathcal{D}$, the rate of polarization $E(K)$ is given by

$$
E(K)=\frac{1}{l} \sum_{i=0}^{l-1} \log _{l} \mathcal{D}_{i} .
$$

It is possible to show that by increasing kernel dimension $l$ to infinity, it is possible to obtain $l \times l$ kernels with rate of polarization arbitrarily close to 1 [2]. Explicit constructions of kernels with high rate of polarization are provided in [4], [5].

2) Scaling exponent: Another crucial property of polarization kernels is the scaling exponent. Let us fix a binary discrete memoryless channel $W$ of capacity $I(W)$ and a desired block error probability $P_{e}$. Given $W$ and $P_{e}$, suppose we wish to communicate at rate $I(W)-\Delta$ using a family of $(n, k)$ polar codes with kernel $K$. The value of $n$ scales as $O\left(\Delta^{-\mu(K)}\right)$, the constant $\mu(K)$ is known as the scaling exponent [10]. The scaling exponent depends on the channel. Unfortunately, the algorithm of its computation is only known for the case of the binary erasure channel [10], [11]. It is possible to show [12], [13] that there exist $l \times l$ kernels $K_{l}$, such that $\lim _{l \rightarrow \infty} \mu\left(K_{l}\right)=2$, i.e. the corresponding polar codes provide an optimal scaling behaviour. Constructions of the kernels with good scaling exponent are provided in [14], [15].

\section{SEARCH ALGORITHM}

In this section we describe an algorithm, which searches for an $l \times l$ kernel with the required partial distance profile. We start from a basic idea and add some improvements, which reduce the overall search space.

\section{A. Basic algorithm}

For given $l \times l$ kernel $K$ we define a sequence of $(l, l-$ $\left.\phi, d_{K}^{(\phi)}\right)$ kernel codes $\mathcal{C}_{K}^{(\phi)}=\langle K[\phi], \ldots, K[l-1]\rangle, \phi \in[l]$, and $\mathcal{C}_{K}^{(l)}$ contains only zero codeword. Suppose we want to obtain an $l \times l$ polarization kernel $K$ with given PDP $\mathcal{D}$. Our idea is to successively construct such a kernel starting from row $K[l-1]$ to row $K[0]$. It is convenient to define sets of candidate rows $\mathbb{M}_{\phi}, \phi \in[l]$, i.e. for each obtained $K$ its rows $K[\phi] \in \mathbb{M}_{\phi}$. The definition of partial distances allows us to initialize these sets as $\mathbb{M}_{\phi}^{(\text {def })}=\left\{v_{0}^{l-1} \mid v_{0}^{l-1} \in \mathbb{F}_{2}^{l}, \operatorname{wt}(v) \geq \mathcal{D}_{\phi}\right\}$.

This approach can be described in terms of depth-first search over candidate rows, which is illustrated in Alg. 1.

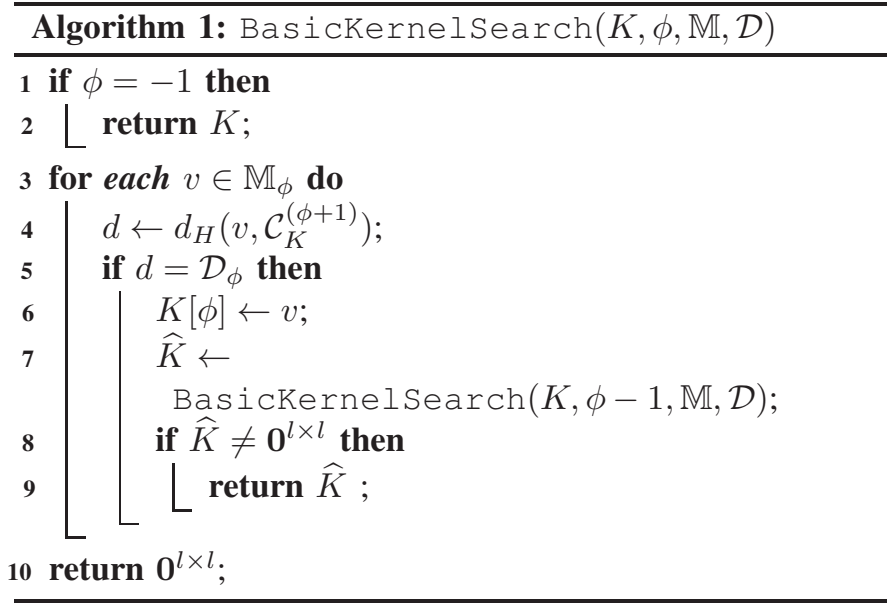

One should start search by calling BasicKernelSearch $\left(\mathbf{0}^{l \times l}, l-\right.$ $\left.1, \mathbb{M}_{\phi}^{(d e f)}, \mathcal{D}\right)$, where $0^{l \times l}$ is a zero $l \times l$ matrix. At phase $\phi$ the algorithm iterates through vectors $v \in \mathbb{M}_{\phi}$ and checks whether $d_{H}\left(v, \mathcal{C}_{K}^{(\phi+1)}\right)=\mathcal{D}_{\phi}$. If such a row is found, the algorithm sets $K[\phi] \leftarrow v$, proceeds to phase $\phi-1$ and starts new search over rows $v \in \mathbb{M}_{\phi-1}$. If there are no such rows, we return to phase $\phi+1$ and continue iterating through $v \in \mathbb{M}_{\phi+1}$. The algorithm traverses the recursion tree until a kernel $K$ with required PDP $\mathcal{D}$ is obtained or zero matrix $\mathbf{0}^{l \times l}$ is returned.

\section{B. Evaluation of partial distances}

Let $A_{K}^{(\phi)}$ and $A_{K}^{(\phi)}(v)$ be weight distributions of $\mathcal{C}_{K}^{(\phi)}$ and $\operatorname{coset} \mathcal{C}_{K}^{(\phi)} \oplus v$, respectively. Observe that

$$
A_{K}^{(\phi-1)}=A_{K}^{(\phi)}+A_{K}^{(\phi)}(K[\phi-1]) .
$$

Equation (4) implies that to obtain all partial distances one can compute the weight distribution of all kernel codes. A good survey of weight distribution computation algorithms can be found in [16] together with a new method based on characteristic vector representation of linear codes.

In this work, for $\phi \geq l / 2$, we compute partial distance directly by definition (1). This allows us to stop the computation of partial distance $d_{H}\left(v, \mathcal{C}_{K}^{(\phi+1)}\right)$ once the row with weight less than $\mathcal{D}_{\phi}$ is occurred, which quickly prunes unsuitable rows.

Let $\overline{\mathcal{C}}_{K}^{(\phi)}$ be dual code of the kernel code $\mathcal{C}_{K}^{(\phi)}$. For phases $\phi<l / 2$ equation (4) enables us to compute $d_{H}\left(K[\phi-1], \mathcal{C}_{K}^{(\phi)}\right)$ by calculation of weight distribution of codes $\overline{\mathcal{C}}_{K}^{(\phi)}, \overline{\mathcal{C}}_{K}^{(\phi-1)}$, and application of MacWilliams identities.

\section{Restriction of candidate rows}

Proposition 1 ([11]). Let $K$ and $\widehat{K}$ be $l \times l$ polarization kernels, where $\widehat{K}$ is obtained from $K$ by adding row $i$ to row $j$ with $j<i$, then $\mathcal{C}_{\widehat{K}}^{(i)}=\mathcal{C}_{K}^{(i)}$ for all $i \in[l]$. Naturally, $E(\widehat{K})=E(K)$ and $\mu(\widehat{K})=\mu(K)$.

Proposition 1 implies that any kernel $K$ can be transformed by addition of row $i$ to row $j$ with $j<i$ into kernel $\widehat{K}$ with $\mathcal{D}_{i}=\operatorname{wt}(\widehat{K}[i]), i \in[l]$, and same polarization properties as 
$K$. Therefore, without loss of generality, the sets of candidate rows can be defined as

$$
\mathbb{M}_{\phi}^{(r)}=\left\{v_{0}^{l-1} \mid v_{0}^{l-1} \in \mathbb{F}_{2}^{l}, \operatorname{wt}(v)=\mathcal{D}_{\phi}\right\} .
$$

Note that one can further restrict $\mathbb{M}_{\phi}$ to construct more specific kernels, as it was done in [14], but in this paper we consider the candidate sets (5) only.

Despite the noticeable restriction (5) of $\mathbb{M}_{\phi}$, function $B a$ sicKernelSearch is still infeasible for large $l$. In the next sections we modify this algorithm to reduce the search space.

\section{Syndrome computation}

Let $H_{K}^{(\phi)}$ be a parity-check matrix of the kernel code $\mathcal{C}_{K}^{(\phi)}$. Observe that if vectors $v$ and $v^{\prime}$ are from the same coset, i.e. $v H_{K}^{(\phi)}=v^{\prime} H_{K}^{(\phi)}$, then, $d_{H}\left(v, \mathcal{C}_{K}^{(\phi)}\right)=d_{H}\left(v^{\prime}, \mathcal{C}_{K}^{(\phi)}\right)$. At each phase $\phi$ we propose to store the set $\mathbb{S}_{\phi}$ of syndromes $v H_{K}^{(\phi+1)}$ of cosets $\mathcal{C}_{K}^{(\phi+1)} \oplus v$, for which the partial distance $d_{H}\left(v, \mathcal{C}_{K}^{(\phi+1)}\right)$ has already been computed. Thus, if for some row $v^{\prime}$ its syndrome $v^{\prime} H_{K}^{(\phi)} \in \mathbb{S}_{\phi}$, then $v^{\prime}$ can be skipped in the loop at lines 3,9 of Alg 1 .

\section{E. Reducing the search space}

Let $\mathcal{C}_{K, v}^{(\phi)}$ denote the code $\langle v, K[\phi], \ldots, K[l-1]\rangle$. We say that $l \times l$ binary polarization kernels $K$ and $\hat{K}$ are equivalent if there exists an $l \times l$ permutation matrix $P$ such as $\hat{K}=K P$. This definition implies that the kernel codes $\mathcal{C}_{K}^{(i)}$ and $\mathcal{C}_{\hat{K}}^{(i)}, i \in$ $[l]$, are also equivalent. We propose to compute $d_{H}\left(v^{\prime}, \mathcal{C}_{K}^{(\phi+1)}\right)$ only if code $\mathcal{C}_{K, v}^{(\phi+1)}$ is not equivalent to all other codes $\mathcal{C}_{\widehat{K}, \widehat{v}}^{(\phi)}$ already arisen at phase $\phi$ of Alg. 1.

Unfortunately, to the best of our knowledge, there are no simple algorithms for testing code equivalence. It was shown in [17] that code equivalence problem can be reduced to the graph isomorphism problem in polynomial time. The support splitting algorithm [18] can be used to find a permutation between equivalent codes. Code equivalence test based on canonical augmentation is proposed in [19], [20].

The code equivalence problem is hard to decide. Instead of this, we propose to consider the codes $\mathcal{C}_{K, v}^{(\phi)}$ with unique weight distribution $A_{K}^{(\phi+1)}(v)$ of the $\operatorname{coset} \mathcal{C}_{K}^{(\phi+1)} \oplus v$. Observe that this distribution can be directly obtained while computing of the partial distance $d_{H}\left(v, \mathcal{C}_{K}^{(\phi)}\right)$. Certainly, nonequivalent codes may have the same weight distribution, therefore, if we use this modification, the algorithm is not guaranteed to find a kernel with given PDP even if it exists.

\section{F. Proposed search algorithm}

Alg. 2 presents a proposed search algorithm for large polarization kernels. One should start search by calling KernelSearch $\left(\mathbf{0}^{l \times l}, l-1, \mathbb{M}_{\phi}^{(r)}\right)$. We assume that the variables $\mathbb{K}$ and $\mathbb{W}$ are global. We also modified the basic algorithm to collect several polarization kernels with PDP $\mathcal{D}$ into the set $\mathbb{K}$. This set is used in Section $\mathbb{V}$ The set $\mathbb{W}_{\phi}$ is a set of weight distributions $A_{K}^{(\phi+1)}(v)$. We used universal hashing [21] for efficient comparison of weight distributions.

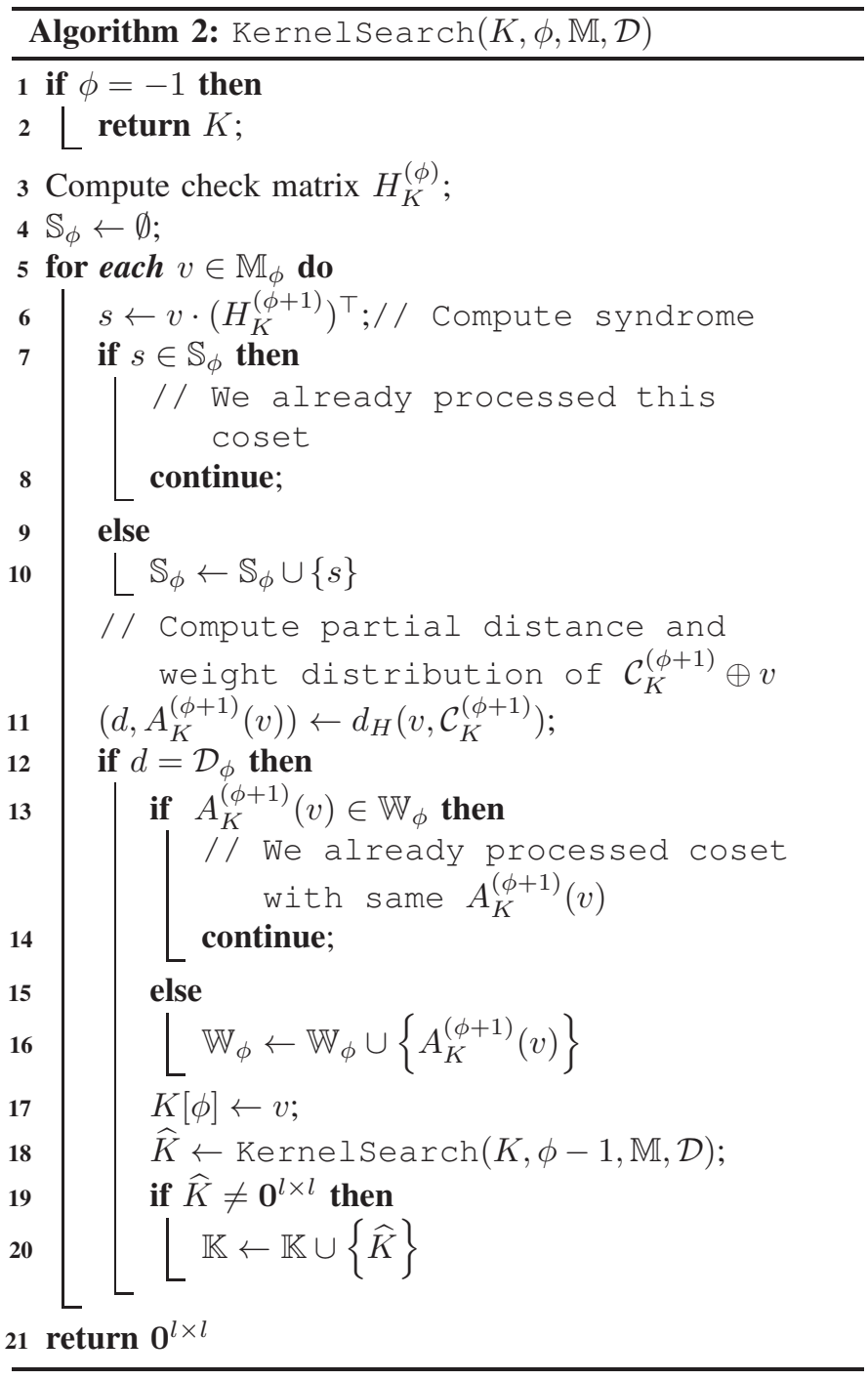

\section{MAXIMIZING RATE OF POLARIZATION}

In this section we describe a method of polarization kernel search, which in many cases improves the lower bound on the maximal rate of polarization $E_{l}$ achievable by $l \times l$ polarization kernel, i.e. $E_{l}=\max _{K \in \mathbb{F}_{2}^{l \times l}} E(K)$. Lower and upper bounds on $E_{l}$ for some $l$ were derived in [2], [4] and [5] together with explicit kernels achieving lower bounds.

\section{A. Search for good partial distance profiles}

According to [2, Corollary 16], maximization of $E_{l}$ can be done by considering only kernels with nondecreasing partial distances, i.e. $\mathcal{D}_{\phi} \leq \mathcal{D}_{\phi+1}$ for $\phi \in[l-1]$.

Proposition 2 (Minimum Distance and Partial Distance [2]). Let $\mathcal{C}_{0}$ be a $\left(n, k, d_{0}\right)$ binary linear code. Let $g$ be an length- $n$ vector with $d_{H}\left(g, \mathcal{C}_{0}\right)=d_{2}$. Let $\mathcal{C}_{1}$ be the $\left(n, k+1, d_{1}\right)$ linear code obtained by adding the vector $g$ to $\mathcal{C}_{0}$, i.e., $\mathcal{C}_{1}=\left\langle g, \mathcal{C}_{0}\right\rangle$. Then $d_{2}=\min \left(d_{0}, d_{1}\right)$.

Consider $l \times l$ kernel $K$ with PDP $\mathcal{D}$. Proposition 2 implies that the minimum distance $d_{K}^{(\phi)}$ of kernel codes $\mathcal{C}_{K}^{(\phi)}, \phi \in[l]$ is 
TABLE I: Polarization properties of the kernels with the best rate of polarization

\begin{tabular}{|c|c|c|c|c|c|c|c|}
\hline$l$ & [2], $E$ & [4], $E$ & \multicolumn{5}{|c|}{ Proposed } \\
\hline 17 & 0.49175 & & 0.49361 & 3.57316 & $1,1,2,2,2,3,4,4,4,5,6,7,8,8,8,8,16$ & 1250 & 6938 \\
\hline 18 & 0.48968 & 0.49521 & 0.50052 & 3.52842 & $1,2,2,2,2,2,4,4,4,6,6,6,6,8,8,10,10,12$ & 2946 & 17414 \\
\hline 20 & 0.49659 & & 0.50617 & 3.43827 & $1,2,2,2,2,2,4,4,4,4,6,6,8,8,8,8,8,8,12,16$ & 4894 & 22578 \\
\hline 21 & 0.48705 & 0.49604 & 0.50868 & 3.37385 & $1,2,2,2,2,2,4,4,4,4,6,6,6,6,8,8,10,10,10,14,14$ & 10978 & 39630 \\
\hline 22 & 0.49445 & 0.50118 & 0.51181 & 3.3530 & $1,2,2,2,2,2,4,4,4,4,6,6,6,6,8,8,8,10,10,10,12,20$ & 18120 & 70430 \\
\hline 25 & 0.50040 & 0.50608 & 0.51683 & 3.28057 & $1,2,2,2,2,2,4,4,4,4,4,6,6,6,8,8,8,8,8,10,12,12,12,16,18$ & 40566 & 164943 \\
\hline 26 & 0.50470 & & 0.51921 & 3.25551 & $1,2,2,2,2,2,4,4,4,4,4,6,6,6,6,8,8,8,10,10,10,12,12,12,14,24$ & 54848 & 191819 \\
\hline 27 & 0.50836 & & 0.51935 & 3.27814 & $1,2,2,2,2,2,4,4,4,4,4,6,6,6,6,8,8,8,8,10,10,10,12,12,14,14,24$ & 93764 & 327428 \\
\hline
\end{tabular}

given by $\min _{\phi \leq i<l} \mathcal{D}_{i}$. Let $d[n, k]$ be a best known minimum distance of $(n, k)$ linear code [22]. Thus, we can apply bound $d[l, l-\phi]$ on kernel codes $\mathcal{C}_{K}^{(\phi)}$, which results in the restriction on partial distance $\mathcal{D}_{\phi} \leq d[l, l-\phi]$.

Lemma 1. ([5. Lemma 4]) Let $\mathcal{D}_{0}, \mathcal{D}_{1}, \ldots, \mathcal{D}_{l-1}$ be a nondecreasing partial distance profile of a kernel of size l. If $\mathcal{D}_{1}=2$, then $\mathcal{D}_{i}$ is even for all $i \geq 1$.

Lemma 2. ([5. Lemma 5]) Let $\mathcal{D}_{0}, \mathcal{D}_{1}, \ldots, \mathcal{D}_{l-1}$ be a nondecreasing partial distance profile of a kernel $K$ of size l. Then, for $0 \leq i<l$, we have $\sum_{i^{\prime}=i}^{l} 2^{l-i^{\prime}} \mathcal{D}_{i^{\prime}} \leq 2^{l-i} l$.

To improve $E_{l}$, we propose the approach similar to those described in [5]. Namely, we generate nondecreasing PDPs $\mathcal{D}$ with $\mathcal{D}_{\phi} \leq d[l, l-\phi]$ satisfying Lemmas 1, 2] and which are LP-valid [4]. We also require that $\frac{1}{l} \sum_{i=0}^{l-1} \log _{l} \mathcal{D}_{i}$ should be larger than existing lower bounds on $E_{l}$. Then, we run Alg. 2 for all valid $\mathcal{D}$. Due to the tremendous search space, for some PDP $\mathcal{D}$ the algorithm may not produce a kernel for a long time. In this case, it should be terminated. Nevertheless, during the computer search we observed that if a kernel with given PDP exists, Alg. 2 often returns it quickly (in a few seconds). This is the advantage of depth-first search, which allows us to perform maximization of $E_{l}$.

\section{B. Results}

Table \presents the parameters of the kernels obtained with the proposed approach. The proposed kernels can be found in Appendix, Fig 3 It can be seen that the proposed kernels have better rate of polarization compared with the ones proposed in [2] and [4], except for $l=24$.

We also report the processing complexity (measured as a number of addition and comparison operations) of the proposed kernels for Viterbi-based processing algorithm [23] and the recently proposed recursive trellis processing algorithm (RTPA) [6], [7]. It can be seen that the complexity of RTPA is significantly lower compared with Viterbi processing. Note that we did not optimize the processing complexity of the proposed kernels, like it was done in [24] to reduce the complexity of Viterbi processing algorithm of $\mathrm{BCH}$ kernels.

We did not try to minimize the scaling exponent of the kernels of considered sizes. We conjecture that it can be further decreased.

\section{KERNELS WITH LOW PROCESSING COMPLEXITY}

Although the polarization kernels from Table \ have excellent polarization properties, their practical usage is limited due to the tremendous processing complexity. We propose to consider polarization kernels with degraded polarization properties and non-monotonic partial distances. This is motivated by fact that the trellis state complexity of an $(n, k, d)$ linear code is lower bounded. That is, by degrading the rate of polarization and permuting the partial distances we reduce the minimal distance of the corresponding kernel codes, thus reducing the lower bound on trellis state complexity. For example, such kernels of size 16 and 32 were proposed in [14] and [25].

We propose to use the algorithm given in Section IV-A to obtain several PDPs corresponding to a reduced rate of polarization. Then we permute some PDP entries and try to construct the corresponding kernels using Alg. 2

The results of complexity minimization are presented in Table III. The proposed kernels can be found in Appendix, Fig 4 It can be seen that by degrading polarization properties we can significantly reduce the processing complexity. For instance, $E\left(K_{27}^{*}\right)=0.51935$ and its RTPA complexity is 93764 operations, whereas $E\left(K_{27}\right)=0.49720$ and its processing complexity is 998 operations, which is 94 times lower.

\section{NUMERIC RESULTS}

Several mixed-kernel (MK) polar subcodes with obtained kernels were constructed. Their performance was investigated for the case of AWGN channel with BPSK modulation. The sets of frozen symbols were obtained by the method proposed in [26]. For MK polar subcodes we used different order of kernels and present the results for the best one.

Fig. 1 illustrates the performance of different $(768,384)$ polar codes decoded by successive cancellation list (SCL) algorithm [27] with list size $L$. We report the results for randomized MK polar subcodes [28], [26] with the proposed $K_{24}$ and $K_{24}^{*}$ kernels, $2^{t} \times 2^{t}$ Arikan matrix $F_{t}=\left(\begin{array}{ll}1 & 0 \\ 1 & 1\end{array}\right)^{\otimes t}$. and $3 \times 3$ kernel $T_{3}$ introduced in [9]. We also include the results for descending (DES) asymmetric construction with 16bit CRC [29] and improved hybrid design [30] of MK polar codes together with the performance of randomized chained polar subcodes [31]. It can be seen that polar codes with 
TABLE II: Polarization properties of kernels which admit low complexity processing

\begin{tabular}{|c|c|c|c|c|c|c|}
\hline$l$ & $E$ & $\mu$ & Partial distances & \multicolumn{2}{|c|}{ Processing complexity } \\
\cline { 5 - 7 } & & & RTPA & Viterbi \\
\hline 18 & 0.49521 & 3.64809 & $1,2,2,4,2,2,2,4,4,6,4,6,8,8,8,8,8,16$ & 1000 & 6507 \\
\hline 20 & 0.49943 & 3.64931 & $1,2,2,4,2,4,2,2,4,4,6,8,8,8,4,8,12,8,8,16$ & 478 & 5632 \\
\hline 24 & 0.50291 & 3.61903 & $1,2,2,4,2,4,2,4,6,2,4,4,8,8,12,4,4,8,8,12,12,8,16,16$ & 365 & 6320 \\
\hline 27 & 0.49720 & 3.76596 & $1,2,2,4,2,2,4,4,6,2,4,4,6,6,8,8,10,12,4,4,8,8,12,12,8,16,16$ & 998 & 14905 \\
\hline 32 & 0.52194 & 3.42111 & $1,2,2,4,2,4,2,4,6,8,2,4,6,8,4,6,8,12,4,8,12,16,4,8,12,16,8,16,8,16,16,32$ & 526 & 13608 \\
\hline
\end{tabular}

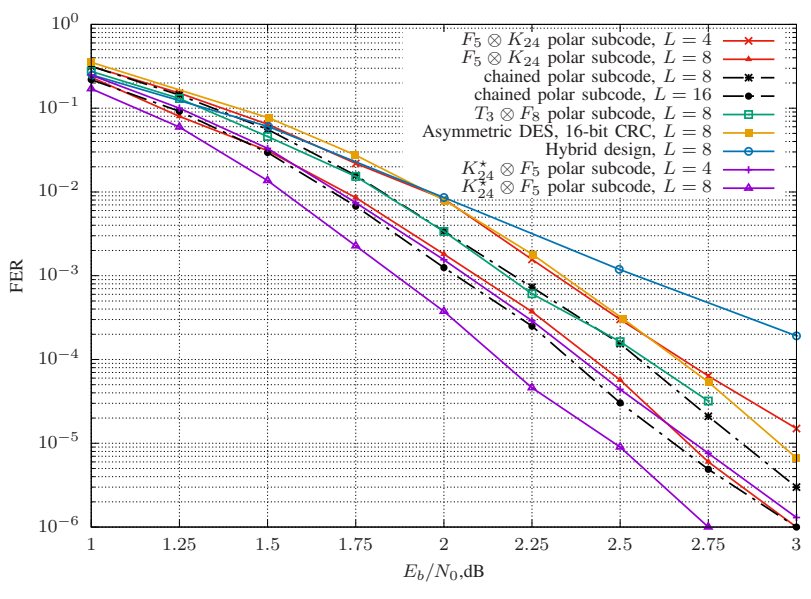

Fig. 1: Performance of $(768,384)$ polar codes

$F_{5} \otimes K_{24}$ polarizing transform under SCL decoding with $L=8$ outperforms chained and $T_{3} \otimes F_{8}$ polar subcodes, asymmetric DES and hybrid construction under SCL with the same list size. Moreover, it provides almost the same performance as chained polar subcode decoded with $L=16$. Observe that despite of having degraded polarization properties (compared with $K_{24}^{*}$ kernel), kernel $K_{24}$ still provides a noticeable performance gain compared with other code constructions.

It can be seen that due to excellent polarization properties of $K_{24}^{*}$, MK polar code with $K_{24}^{*} \otimes F_{5}$ under SCL with $L=4$ provides the same performance as $F_{5} \otimes K_{24}$ with $L=8$.

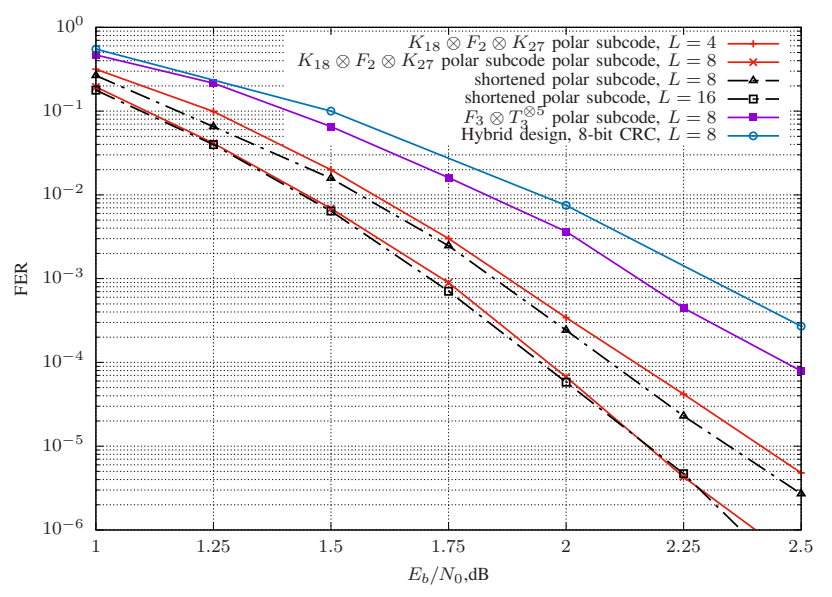

Fig. 2: Performance of $(1944,972)$ polar codes

Fig. 2 presents the performance of various $(1944,972)$ polar codes. It can be seen that polar subcode with $K_{18} \otimes F_{2} \otimes K_{27}$ polarizing transform under SCL decoding with $L=4$ provides significant performance gain compared to MK polar subcode with $F_{3} \otimes T_{3}^{\otimes 5}$ polarizing transform and hybrid design wit 8-bit CRC [9]. Moreover, $K_{18} \otimes F_{2} \otimes K_{27}$ polar subcode with $L=8$ has the same performance as shortened polar subcode decoded with $L=16$. We should also point out that despite of $E\left(K_{18}\right)<0.5$ and $E\left(K_{27}\right)<0.5$, the resulting code achieves significant performance gain compared with shortened polar code with $F_{11}$ having $E\left(F_{11}\right)=0.5$. In addition, we conjecture that polarization properties of kernels with different size can not be properly compared. That is, kernels $K$ with $l \neq 2^{t}$ and even $E(K)<0.5$ should be studied and can be considered in mixed kernel polar codes.

More results on performance and complexity comparison of polar codes with the proposed kernels can be found in [6].

\section{CONCLUSION AND OPEN PROBLEMS}

In this paper a novel search method for large polarization kernels with given partial distance profile was proposed. This algorithm is based on depth-first search over sets of candidate rows. The proposed algorithm was used to improve lower bounds on the maximal rate of polarization of sizes $17 \leq l \leq 27$.

We also demonstrated application of the proposed method for finding kernels which admit low complexity processing by the recursive trellis processing algorithm. Numerical results demonstrate the advantage of polar codes with the obtained kernels compared with shortened polar codes with Arikan kernel and polar codes with small kernels.

In the following we outline multiple open problems with large kernels:

- Tight bounds on partial distances. There is a significant gap between lower and upper bounds on $E_{l}$ for large $l$. Moreover, we have almost no bounds for non-monotonic partial distances, except LP bounds.

- The problem of scaling exponent minimization remains unsolved. It is unknown whether the proposed method can be extended to explicitly solve it.

- Processing complexity. For some PDP the proposed algorithm can produce kernels which admit low complexity processing by RTPA. However, we do not know how to explicitly minimize a processing complexity for kernels with given PDP.

\section{ACKNOWLEDGEMENT}

This work is partially supported by the Ministry of Science and Higher Education of Russian Federation, passport of goszadanie no. 2019-0898. 


\section{APPENDIX}

Fig. 3 presents polarization kernels with the best rate of polarization, obtained in Section IV] Fig. 4 presents polarization kernels which admit low complexity processing, obtained in Section $\mathrm{V}$

\section{REFERENCES}

[1] E. Arıkan, "Channel polarization: A method for constructing capacityachieving codes for symmetric binary-input memoryless channels," IEEE Transactions on Information Theory, vol. 55, no. 7, pp. 3051-3073, July 2009.

[2] S. B. Korada, E. Sasoglu, and R. Urbanke, "Polar codes: Characterization of exponent, bounds, and constructions," IEEE Transactions on Information Theory, vol. 56, no. 12, pp. 6253-6264, December 2010.

[3] A. Fazeli, S. H. Hassani, M. Mondelli, and A. Vardy, "Binary linear codes with optimal scaling: Polar codes with large kernels," in Proceedings of IEEE Information Theory Workshop, 2018.

[4] N. Presman, O. Shapira, S. Litsyn, T. Etzion, and A. Vardy, "Binary polarization kernels from code decompositions," IEEE Transactions On Information Theory, vol. 61, no. 5, May 2015.

[5] H.-P. Lin, S. Lin, and K. A. Abdel-Ghaffar, "Linear and nonlinear binary kernels of polar codes of small dimensions with maximum exponents," IEEE Transactions On Information Theory, vol. 61, no. 10, October 2015.

[6] P. Trifonov, "Recursive trellis processing of large polarization kernels," in Proceedings of IEEE International Symposium on Information Theory, 2021.

[7] — "Trellis-based decoding techniques for polar codes with large kernels," in Proceedings of IEEE Information Theory Workshop, 2019, invited paper.

[8] N. Presman, O. Shapira, and S. Litsyn, "Mixed-kernels constructions of polar codes," IEEE Journal on Selected Areas in Communications, vol. 34, no. 2, pp. 239-253, Feb 2016.

[9] V. Bioglio, F. Gabry, I. Land, and J. C. Belfiore, "Multi-kernel polar codes: Concept and design principles," IEEE Transactions on Communications, vol. 68, no. 9, pp. 5350-5362, 2020.

[10] S. H. Hassani, K. Alishahi, and R. Urbanke, "Finite-length scaling for polar codes," IEEE Transactions On Information Theory, vol. 60, no. 10, October 2014

[11] A. Fazeli and A. Vardy, "On the scaling exponent of binary polarization kernels," in Proceedings of 52nd Annual Allerton Conference on Communication, Control and Computing, 2014, pp. 797 - 804.

[12] H. Pfister and R. Urbanke, "Near-optimal finite-length scaling for polar codes over large alphabets," in Proceedings of IEEE International Symposium on Information Theory, 2016.

[22] M. Grassl, "Bounds on the minimum distance of linear codes and quantum codes," Online available at http://www.codetables.de. 2007, accessed on 2021-01-21.
[13] A. Fazeli, H. Hassani, M. Mondelli, and A. Vardy, "Binary linear codes with optimal scaling: Polar codes with large kernels," IEEE Transactions on Information Theory, 2021.

[14] G. Trofimiuk and P. Trifonov, "Construction of binary polarization kernels for low complexity window processing," in Proceedings of IEEE Information Theory Workshop, 2019.

[15] H. Yao, A. Fazeli, and A. Vardy, "Explicit polar codes with small scaling exponent," in Proceedings of IEEE International Symposium on Information Theory, 2019.

[16] I. Bouyukliev, S. Bouyuklieva, T. Maruta, and P. Piperkov, "Characteristic vector and weight distribution of a linear code," Cryptography and Communications, vol. 46, no. 4, pp. 1936-2455, 2020.

[17] E. Petrank and R. M. Roth, "Is code equivalence easy to decide?" IEEE Transactions on Information Theory, vol. 43, no. 5, pp. 1602-1604, 1997.

[18] N. Sendrier, "Finding the permutation between equivalent linear codes: the support splitting algorithm," IEEE Transactions on Information Theory, vol. 46, no. 4, pp. 1193-1203, 2000.

[19] I. G. Bouyukliev, "About the code equivalence," Advances in Coding Theory and Cryptography, pp. 126-151.

[20] I. Bouyukliev, S. Bouyuklieva, and S. Kurz, "Computer classification of linear codes," arXiv:2002.07826. 2020.

[21] M. Thorup, "High speed hashing for integers and strings," arXiv:1504.06804 2020.

[23] H. Griesser and V. R. Sidorenko, "A posteriory probability decoding of nonsystematically encoded block codes," Problems of Information Transmission, vol. 38, no. 3, 2002.

[24] E. Moskovskaya and P. Trifonov, "Design of BCH polarization kernels with reduced processing complexity," IEEE Communications Letters, vol. 24, no. 7, pp. 1383-1386, July 2020.

[25] G. Trofimiuk and P. Trifonov, "Window processing of binary polarization kernels," IEEE Transactions on Communications, 2021, to appear.

[26] P. Trifonov, "On construction of polar subcodes with large kernels," in Proceedings of IEEE International Symposium on Information Theory, Paris, France, 2019.

[27] I. Tal and A. Vardy, "List decoding of polar codes," IEEE Transactions On Information Theory, vol. 61, no. 5, pp. 2213-2226, May 2015.

[28] P. Trifonov and G. Trofimiuk, "A randomized construction of polar subcodes," in Proceedings of IEEE International Symposium on Information Theory. Aachen, Germany: IEEE, 2017, pp. 1863-1867.

[29] A. Cavatassi, T. Tonnellier, and W. J. Gross, "Asymmetric construction of low-latency and length-flexible polar codes," in ICC 2019 - 2019 IEEE International Conference on Communications (ICC), 2019, pp. $1-6$.

[30] V. Bioglio, I. Land, and C. Condo, "Improved hybrid design of polar codes and multi-kernel polar codes," in 2019 IEEE International Symposium on Information Theory (ISIT), 2019, pp. 1947-1951.

[31] P. Trifonov, "Randomized chained polar subcodes," in Proceedings of IEEE Wireless Communications and Networking Conference Workshops. Barcelona, Spain: IEEE, 2018, pp. 292-297. 


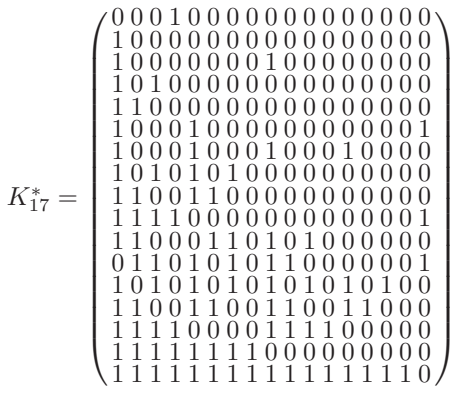

110000000000000000000 10000010000000000000 10000100000000000000 10010000000000000000 10100000000000000000 11000000000000000000 10010100000010000000 11000100000000001000 100010111000000000000 110 10111001001000000000 11011010100000000000 10110100100010001010 01101100101000001100 10101010101010100000 00111111110000000000 11001100110011000000 11110000111100000000

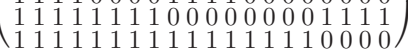

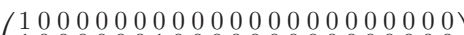
10000001000000000000000 10001000000000000000000 10010000000000000000000 10100000000000000000000

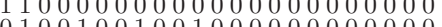
1.

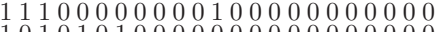

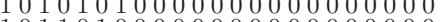

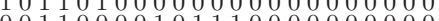
00110000101110000000000 $\begin{array}{llllllllllllllllllllll}1 & 0 & 1 & 0 & 0 & 1 & 0 & 1 & 0 & 0 & 1 & 0 & 0 & 1 & 0 & 0 & 0 & 0 & 0 & 0 & 0 & 0 \\ 1\end{array}$ 11000100010100010000000 11100110100000000000 11001101101000000000100 11111011010000000000000 10011011101000101001000 00101101111001001010000 11001110110010001100000 11110000111100001110001

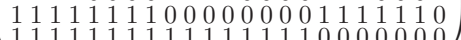

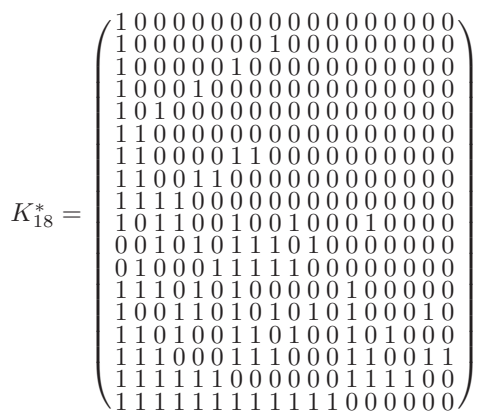

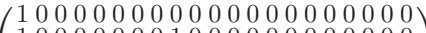
10000000100000000000000

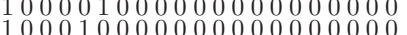

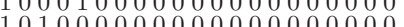
101000000000000000000 110000000000000000000

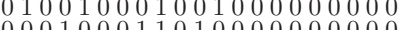

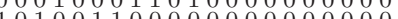
111100000000000000000

$K_{21}^{*}=$ 111100000000000000000 1

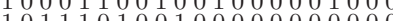

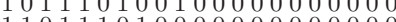

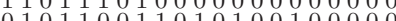
10110011010100100000 101011111000001000000

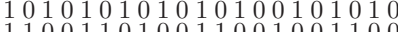
110011010011001001100 111100011100001110000

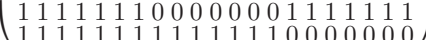

000000000000000 100000000000000010000000 10001000000000000000000000 100000001000000000000000 101000000000000000000000 110000000000000000000000 100010001000100000000000 101000001010000000000000 110000001100000000000000 101010100000000000000000 110011000000000000000000 年 11100000000000000000000

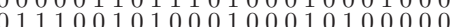
101010101100000011000000 11010100110101000000000 1111001100110000000000 10011001111000000000000 1. 1101010101010 10101010101010 1110000111100001111000 1.1 111111111111111100000000
11000000000000000000 10000001000000000000

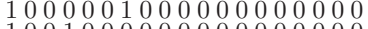
1001000000000000000 10010000000000000000000 1100001100000010000 1000110010000000 1011010000000000000

$K_{19}^{*}=1110100000000000000$ 110011000010000000 1100111000010000000

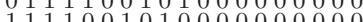
110101011100010100 01001000 1110110011001000000 111000 111000011110000101 1111111100000000110 1000000000000000000000

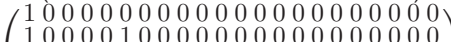

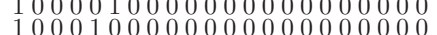

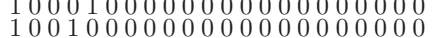
1010000000000000000000 1100000000000000000000 0010110010000000000000 1010100000100000000000 1010010100000000000000 1110001000000000000000

$K_{22}^{*}=$ 1101101000001000000000 0101000001011000010000 1000001100101010000000 1110010011000000000000 0100011000111010001000 1011011010100000100000 $\begin{array}{lllllllllllllllllllllll}0 & 1 & 1 & 1 & 0 & 1 & 1 & 0 & 1 & 1 & 1 & 0 & 0 & 0 & 0 & 0 & 0 & 0 & 0 & 0 & 0\end{array}$ 1110001110110011000000 1111110000111100000000 1111111111000000000011

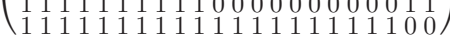
$\left(\begin{array}{llll}1000000000000000000000000 \\ 1\end{array}\right.$ 1.

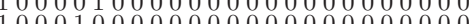

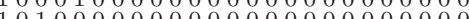
1100000000000000000000000

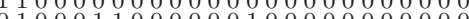
0

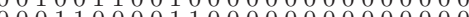
000110001100000000000 1 111100000000000000000000

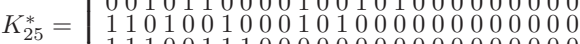
1110011100000000000000000 0101001110000101010000000 0010111101000000010001000 1. 1111000100001100000100000 0111001101110000000000000 1001111111010000001000000 1101010101010101000101010 1110011001100110001001100 1111100001111000001110000

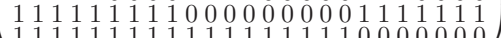

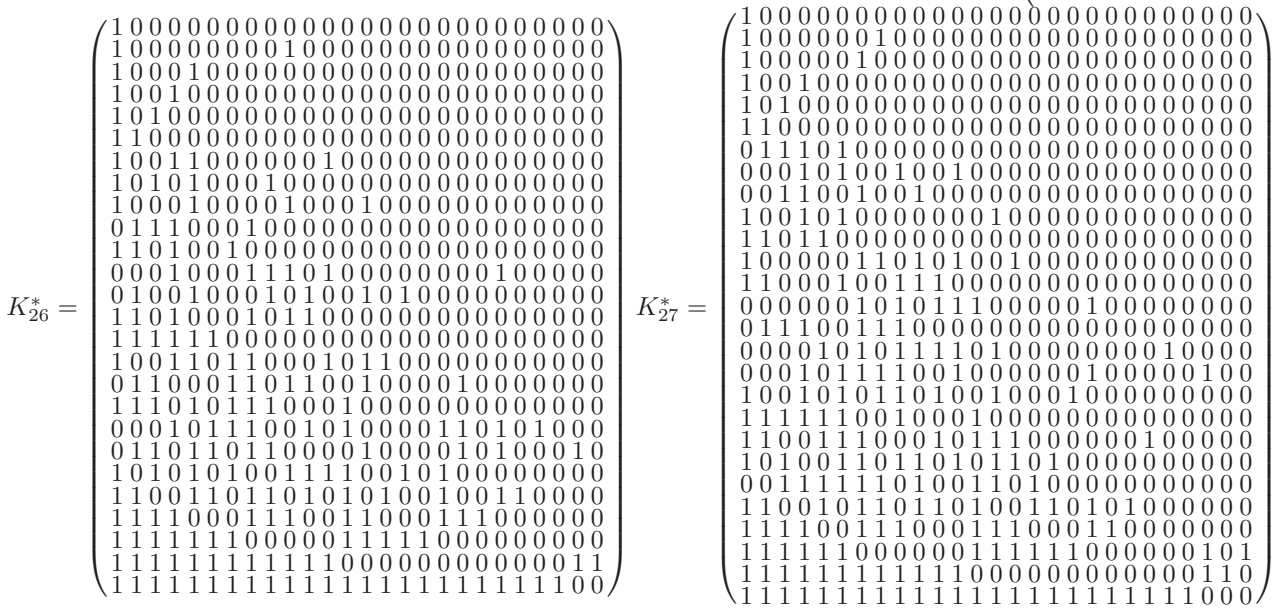

Fig. 3: Polarization kernels with the best rate of polarization 

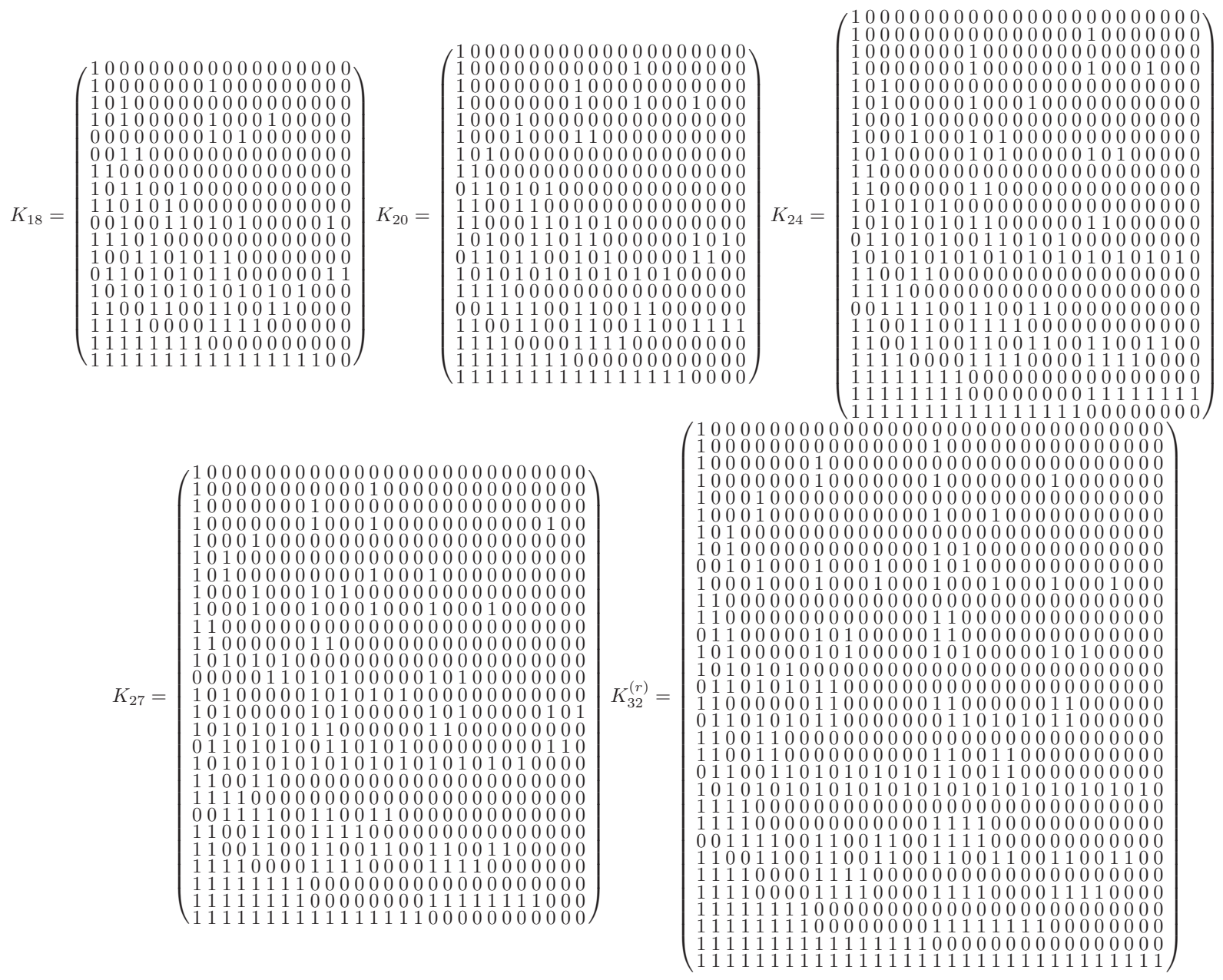

Fig. 4: Polarization kernels which admit low complexity processing 